\title{
Étude expérimentale de cas particuliers : une alternative aux études contrôlées randomisées?
}

\author{
Agnes von Wyl \& Aureliano Crameri \\ Psychotherapie-Wissenschaft 9 (2) 72-73 2019 \\ www.psychotherapie-wissenschaft.info \\ CC BY-NC-ND \\ https://doi.org/10.30820/1664-9583-2019-2-72
}

Mots clés : fondement par les preuves, Étude expérimentale de cas particulier, RCT

Les études expérimentales de cas particuliers sont utilisées de plus en plus souvent dans la recherche en psychothérapie (v. p. ex. l'article de Herrera et al. dans ce cahier). Pour les instituts de formation en psychothérapie se pose la question de savoir si ces derniers représentent une alternative aux études contrôlées randomisées (randomized controlled trials; RCT). Il faut notamment évaluer la valeur qui leur est accordée dans la recherche relative à la psychothérapie fondée sur les preuves.

Les études expérimentales de cas particuliers sont citées par l'APA comme très utiles pour évaluer les interactions causales dans le déroulement de thérapies individuelles; en sus d'autres designs de recherche, elles pourraient ainsi apporter une contribution importante à l'evidence based practice. Elles mettent en œuvre des designs RCT pour prouver la relation de cause à effet d'origine entre une intervention et le résultat de la thérapie (APA, 2006). Chez Chambless et Ollendick (2001), il existe une série d'études expérimentales de cas particuliers à la condition préalable qu'un manuel thérapeutique ou une description sans ambiguité de l'approche de traitement soit utilisé à titre d'équivalent à une RCT.

Mais que contient une étude expérimentale de cas particulier ? Celle-ci peut représenter des relations de cause à effet pour le cas particulier. Dans ce cadre, les effets sont comparés les uns aux autres par rapport au résultat d'une thérapie à au moins deux conditions différentes (p. ex. baseline et intervention). Deux schémas éventuels de design de cas particulier doivent être commentés sur la base des figures 1 et 2. L'effet d'une intervention (B) ainsi que chaque baseline $(\mathrm{A})$ est représenté dans les deux schémas.

Dans le premier schéma (fig. 1), l'importance des symptômes est enregistrée quotidiennement pendant huit jours. À partir du neuvième jour, l'intervention se déroule pendant dix jours. Là aussi, l'importance des symptômes est relevée tous les jours.

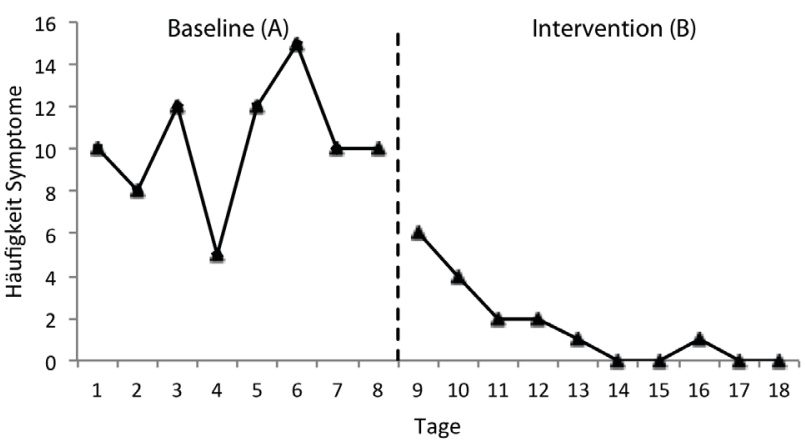

Fig. 1 : design baseline simple

Dans le second schéma (fig. 2), il y a deux phases de comparaison pour l'intervention, à savoir à nouveau le temps précédant la thérapie, mais en sus encore le temps qui vient après. Dans l'exemple présenté par Herrera et al. (dans ce cahier), c'est ce deuxième schéma qui a été appliqué ; les thérapies ont duré entre 8 et 40 sessions.

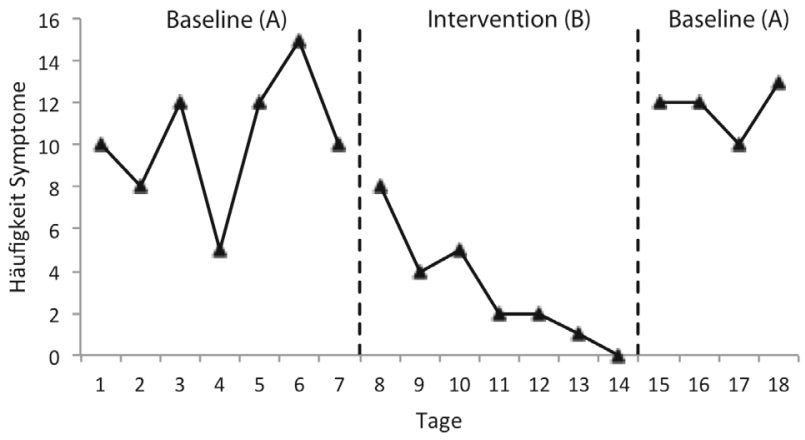

Fig. 2 : design d'inversion

L'exemple suivant doit être présenté comme une variation possible : un certain exercice de thérapie corporelle, qui serait utilisé à partir de la neuvième session dans 
le cadre d'une psychothérapie, pourrait aussi être par exemple défini comme intervention. La psychothérapie sans cet exercice de thérapie corporelle représenterait alors la baseline. Ce design permettrait d'estimer l'effet d'une technique donnée sur le résultat de la thérapie. On pourrait en outre comparer ce design avec des cas de contrôle, c'est-à-dire avec des thérapies dans lesquelles la seule thérapie est appliquée sans intervention supplémentaire.

Il faut en outre tenir compte du fait que des relevés continus de symptômes ou d'autres indicateurs ou facteurs intéressants sont nécessaires pour servir de base à l'évaluation en tant que condition préalable importante pour les études expérimentales de cas particuliers. Cela vaut également pour le relevé de base (voir aussi Kazdin, 2016). Il faut donc que des données soient relevées sur un certain laps de temps pour les trois phases, ce qui veut dire qu'un relevé unique par formulaire avant le début de la thérapie ne suffit pas. Nous renvoyons à la littérature développant plus amplement ce sujet pour la procédure plus précise à suivre pour les études expérimentales de cas particuliers (p. ex. ibidem ainsi que Manolov et al., 2014). On trouvera chez Michiels et Onghena (2018) une description des difficultés que la réalisation d'une étude de cas particulier peut comprendre.

Les études expérimentales de cas particuliers peuvent également, pour les instituts psychothérapeutiques, représenter une possibilité de satisfaire de l'exigence par la recherche d'un fondement par les preuves de son approche psychothérapeutique avec moins de cas qu'une RCT. Il faut cependant être conscient du fait qu' " expérimental » veut également dire que les thérapies doivent être mises en œuvre d'après certains critères préalablement définis. De ce point de vue, l'étude expérimentale de cas particulier ne se différencie pas d'une RCT. Dans une RCT, tous les participants d'un groupe reçoivent une certaine intervention et tous les participants de l'autre groupe une autre. Dans l'étude expérimentale de cas particulier, un patient individuel ou une patiente indi- viduelle à proprement parler reçoit deux interventions différentes ou est soumis à deux différentes conditions, à savoir « aucune intervention » par opposition à « une intervention ».

\section{Littérature}

APA Presidential Task Force on Evidence-Based Practice (2006). Evidence-based practice in psychology. American Psychologist, 61, 271-285. https://doi.org/10.1037/0003-066X.61.4.271

Chambless, D. L. \& Ollendick, T.H. (2001). Empirically supported psychological interventions: controversies and evidence. Annu Rev Psychol, 52, 685-716. https://doi.org/0.1146/annurev.psych.52.1. 68552/1/685

Kazdin, A.E. (2016). Single-case experimental research designs. In A.E. Kazdin (Hrsg.), Methodological issues and strategies in clinical research (S. 459-483). Washington, D. C.: APA. https://doi. org/10.1037/14805-029

Manolov, R., Gast, D.L., Perdices, M. \& Evans, J.J. (2014). Single-case experimental designs: reflections on conduct and analysis. Neuropsychol Rehabil, 24, 634-660. https://doi.org/10.1080/0960 2011.2014.903199

Michiels, B. \& Onghena, P. (2018). Randomized single-case AB phase designs: Prospects and pitfalls. Behavior Research Methods, 51, 1-23. https://doi.org/10.3758/s13428-018-1084-x

\section{Les auteures et auteurs}

Agnes von Wyl, Prof. Dr., est directrice du groupe spécialisé psychologie clinique et psychologie de la santé à la Haute école zurichoise pour les sciences appliquées, département psychologie.

Aureliano Crameri, Dr., est professeur pour les méthodes de recherche et collaborateur scientifique à la Haute école zurichoise pour les sciences appliquées, département psychologie.

\section{Contact}

E-Mail : agnes.vonwyl@zhaw.ch

E-Mail : aureliano.crameri@zhaw.ch 